\title{
PROFESSIONAL COMMUNICATION AS PART OF THE PROFESSIONAL CULTURE OFPROFESSIONALS
}

\author{
Svitlana AMELINA
}

\begin{abstract}
The article is devoted to the culture of professional communication. An attempt to analyze the ways of its formation and development has been made.
\end{abstract}

Key words: culture of professional communication, professional communication, formation, development.

\section{BERUFSKOMMUNIKATION ALS BESTANDTEIL DER BERUFSKULTUR DER FACHLEUTE}

Abstrakt: Es handelt sich im vorliegenden Artikel um Fragen der Kultur der Berufskommunikation. Es wurde ein Versuch der Analyse ihrer Formierungs- und Entwicklungswege gemacht.

Schüsselwörter: Kultur der Berufskommunikation, Berufskommunikation, Formierung, Entwicklung.

\section{Themenaktualität}

Zur Berufskultur der Fachleute gehört u.a. Berufskommunikation, die durch verschiedene Kommunikationsformen ausgedrückt wird. Die Studenten können sich Kenntnisse, Fähigkeiten und Fertigkeiten der Berufs- und Geschäftskommunikation nur dann aneignen, wenn man das kommunikative Herangehen an die Formierung der Kultur der Berufskommunikation bei den Studenten anwendet. Das kommunikative Herangehen beim Unterricht in verschiedenen Fächern (Pflicht-, Pflichtwahl- und Wahlfächern) kann einer der Wege der Formierung der Kultur der Berufskommunikation sein.

Kommunikative Orientierung des Lernpr-zesses ist mit axiologischer Ausrichtung und Handlungsorientierung $\mathrm{zu}$ verbinden. D-durch wird der Lernprozess in den sozialen, kulturellen und wertenden Kontext des Inhalts der beruflichen Ausbildung der Fachleute an den Hochschulen eingeschlossen.

Auf solche Weise werden die Studenten in die Bereiche der berufsorientierter Kultur, Basiskultur und Kultur einer bestimmten sozialen Gruppe herangezogen. Durch eine solche Kombination werden die Voraussetzungen für die Vorbereitung der Fachleute geschaffen, die zur kreativen Selbstverwirklichung in der zukünftigen beruflichen Tätigkeit fähig sind.

\section{Analyse der letzten Forschungen und Veröffentlichungen zum Thema}

Der Begriff „Kommunikation” (vom lateinischen „communico” - ,ich unterhalte mich") wurde von Philosophen, Sprachwissenschaftlern und Psychologen traditionell als ein Begriff, der nah zu den Begriffen „Unterhaltung”, „Umgang”, „Verständigung” ist, betrachtet [Советский энциклопедический словарь 1982: 617]. In der letzten Zeit wird immer mehr die Meinung über die Kommunikation als über den inhaltlichen Aspekt der sozialen Wechselwirkung verbreitet [Matwijenko 2006; Molokanow 1995; Mussatow 1998; Winkel 1980]. Ohne Kommunikation ist die Berufskultur kaum vorzustellen. Die Berufskultur schließt unter vielen Aspekten auch kommunikative Interaktion ein.

Ziel des Artikels ist es, das Problem der kommunikativen Interaktion bei der Formierung der Berufskultur bei den Studenten der Universitäten und Hochschulen zu betrachten.

\section{Grundtext}

Indem wir die Definition der Kommunikation von M.Kagan: „Kommunikationsprozess ist die Übergabe der bestimmten Mitteilungen" berücksichtigen [Kagan 1988:56], halten wir es für notwendig, den Wunsch nach Berufskommunikation bei den zukünftigen Fachleuten hervorzurufen und das bewußte Streben zum Informationsaustausch mit dem Partner (Mitteilung bestimmter Informationen und Entgegennahme der Informationen) zu formen. Zugleich teilen wir auch die Ansichten der Wissenschaftler, die meinen, dass die kommunikative Wechselwirkung bei der Berufskommunikation über den Rahmen der einfachen Informationsaustausch hervorgeht. So 
unterscheidet

W.Semytschenko

solche

Funktionen der

kommunikativen

Wechselwirkung:

- kontaktknüpfend (Erreichung von den Gesprächspartnern des Zustandes der Bereitschaft zur Unterhaltung der Wechselbeziehung während der Interaktion);

- informativ (Mitteilungsaustausch);

- anregend (Ermunterung des Partners zur Aktivität);

- koordinierend (Abstimmung der Handlungen für die Organisation der gemeinsamen Tätigkeit der Partner);

- verständigend (richtiges Verstehen des Sinnes der Miteilung und des Zustandes des Gesprächspartners);

- emotional (zielgerichtetes Hervorrufen der erforderlichen emotionalen Erlebnissen bei dem Partner oder Veränderung des eigenen emotionalen Zustandes mit seiner Hilfe);

- beziehungsbildend (Wahrnehmung eigener Stellung im System der Rollen-, Status-, Geschäfts-, zwischenpersönlichen und anderen Verbindungen in der Gesellschaft);

- beeinflussend (Veränderung des Zustandes oder Benehmens des Partners) [Semytschenko 1999: 38].

Die oben aufgeführte Auflistung der Funktionen der kommunikativen Wechselwirkung betrachtend, kann man die Schlusfolgerung ziehen, dass die Wechselwirkung bei der Berufskommunikation eng mit der kommunikativen Interaktion verbunden ist. Genau so wie die kommunikative Interaktion betrifft die Wechselwirkung bei der Berufskommunikation insbesondere die Formierung der Beziehungen und Suche der Fachleute nach ihrer eigener Stellung in der Gesellschaft sowie ihre Wahrnehmung.

Theoretische Grundlage dafür kann im Prozess des Unterrichts der Geisteswissenschaften geschaffen werden. Sinnvoll wäre z.B. die Einbeziehung der philosophischen Quellen der Kommunikation und die Konzentration auf den Besonderheiten der Rhetorik in verschiedenen historischen Epochen; rhetorischen Lehren von Aristoteles; auf Rednerkunst der Epoche des Hellenismus, deren Techniken auch heutzutage bei der Berufsund Geschäftskommunikation erfolgreich eingesetzt werden; auf Reden und rhetorischen Werken von Cicero; auf Rednerkunst der römischen Epoche; chrislicher Apologetik; spätantiker Rhetorik; östlicher und westlicher
Patriotik. Es soll dabei betont werden, dass die moderne Rednerkunst auf den historischen Erfahrungen beruht. Z.B. sind solche Begriffe aus dem Bereich der Rednerkunst wie „Vorhaben”, „Idee”, „Thema”, „Hypothese”, „Konzept” noch zur antiken Zeit entstanden. Von der altgriechischen und römischen Rhetorik hat man die Struktur des Vortrages mit seinen Hauptteilen (Einführung, Darlegung, Schlussfolgerung) übernommen.

Beim Unterricht von Psychologie ist es wichtig, die Studenten über psychologische Aspekte der Kultur der Berufskommunikation zu unterrichten, darunter auch über die individuelle psychologische Merkmale und Besonderheiten der Persönlichkeit und Formierung der zwischenpersönlichen Beziehungen.

Die Studenten können auch über das kommunikative Verhalten in verschiedenen Kommunikationssituationen, über Mittel zur Selbstbeherrschung und kontrolle sowie zur Vermeidung von Konflikten unterrichtet werden. Dabei sollten sie auch erfahren, dass der Berufsdialog zum Mittel der Regulierung der Verhältnisse der Streitseiten werden kann. Auf solche Weise werden neue Kenntnisse über Berufskommunikation, Kultur der Berufskommunikation und ihre Komponenten von den Studenten angeeignet.

Außerdem soll die Umsetzung der von den Studenten angeeigneten Kenntnisse in den konkreten Kommunikationssituationen und die Formierung der Fertigkeiten der Sprechtechnik stattfinden, was vorsieht:

- Organisation der Lernkommunikation auf Grund von Subjekt-Subjekt-Verhältnissen;

- Heranziehen der Studenten zur aktiven Teilnahme an den dialogischen Unterrichtsformen;

- Formierung des kommunikativen Verhaltens in verschiedenen Berufskommunikationssituationen;

- Erwerb der Hörfertigkeiten;

- Trainieren der Fertigkeit, den emotionalen Zustand des Gesprächspartners wahrzunehmen;

- Entwicklung der Selbstkontrolle, besonders in den Stresssituationen, und der Fertigkeit, adäquat auf die Oppositionsaussagen zu reagieren;

- Entwicklung der Empathie;

- Entwicklung der toleranter Stellung gegenüber den Kommunikationspartnern; 
- Entwicklung der Fertigkeiten, eigene Position zu begründen, zu verteidigen und zu argumentieren;

- Erwerb der Erfahrungen bei der Wechselwirkung der Teilnehmer am pädagogischen Prozess der Universität oder der Hochschule;

- Entwicklung der Kreativität bei der Suche nach Lösungen in den ungewöhnlichen Kommunikationssituationen.

Kommunikative Fähigkeiten und Fertigkeiten werden integriert trainiert, damit sie von den Studenten in realen Situationen frei eingesetzt werden können. Kommunikative und Handlungsorientierung der Unterrichtseinheiten tragen zur Beseitigung der Unterschiede zwischen der natürlichen Kommunikation in der Gesellschaft und der künstlichen Kommunikation im Unterricht bei.

Durch allgemeine kommunikative Ausrichtung des Unterrichtsprozesses sind alle Komponenten der Kultur der Berufskommunikation zu entwickeln. Zu diesem Zweck soll eine besondere Aufmerksamkeit solchen Organisationsformen der Unterrichtstätigkeit der Studenten gewidmet werden Rollen- und Geschäftsspiele, Diskussionen, Gruppenarbeit, Projektarbeit, Fallstudien. Diese Formen fördern die Formierung der Wert- und Moralorienierungen der zukünftigen Fachleute, weil sie ihre aktive Interaktion miteinander und mit dem Lehrer, gleichberechtigte Partnerverhältnisse, Toleranz und Respekt gegenüber dem Opponenten vorsehen.

Bei der Wahl der Materialien für die kommunikativ orientierten Vorlesungen und Seminare ist es $\mathrm{zu}$ empfehlen, Folgendes $\mathrm{zu}$ berücksichtigen:

- altersbedingte Besonderheiten und Bedürfnisse der Lerner;

- Niveau der Vorkenntnisse der Studenten und ihre kommunikative Erfahrungen;

- Themen-, Begriffs- und Hintergrundinhalt der Informationsabschnitte der Mitteilung;

- Aktualität der didaktischen Materialien für selbständige Arbeit an der fachorientierten
Aufgaben (mündliche und schriftliche Kommunikation).

\section{Schlussfolgerung}

Im Ergebnis der zielgerichteten Arbeit mit Orientierung an die Formierung der Fähigkeiten und Fertigkeiten der kommunikativen Interaktion und der operationstechnologischen Fertigkeiten der zukünftigen Fachleute auf Grund der ausgewählten Mittel (speziell gewählte Übungen, dialogisches Unterrichtsverfahren, Trainings) können die Studenten die Erfahrungen bei der Lösung der Berufsprobleme durch Berufskommunikation erwerben. Dabei lernen sie ihre Position zu begründen und zu argumentieren, den Partner zu überzeugen, eigene Taktik und Strategie des Handelns in Abhängigkeit von der Situation der Berufskommunikation zu entwickeln.

\section{Literatur}

1. Каган М.С. Мир общения: Проблема межсубъектных отношений / М.С. Каган. М.: Политиздат, 1988. -319 с.

2. Матвієнко О.В. Професійне педагогічне спілкування як особлива форма взаємодії викладача і студента у навчально-виховному процесі/ О.В. Матвієнко // Вісник Черкаського університету. - Серія „Педагогічні науки”. Випуск 88. - Черкаси, 2006. - С.95-101.

3. Молоканов М.В. Модели коммуникативного взаимодействия // Вопросы психологии. - 1995. - №5. - С.51-60.

4. Мусатов С.О. Психологічний зміст педагогічної комунікації // Культурологічні та психолого-педагогічні аспекти гуманізації освіти: Науково-методичний збірник/ За ред. Г.О.Балла. - К.: Наукова думка, 1998. - С.1422.

5. Советский энциклопедический словарь. М.: Советская энциклопедия, 1982. - 1600 с.

6. Семиченко В.А. Психологія соціальних відносин / В.А. Семиченко. - К.: Магістр-S, 1999. - $168 \mathrm{c}$.

7. Winkel R. Die kritisch-kommunikative Didaktik. In: Westermanns Päd. Beiträge 32 (1980) - S.200-204. 